Mots. Les langages du politique

Trente ans d'étude des langages du politique

(1980-2010)

\title{
Discours institutionnels.Perspectives pour les sciences de la communication
}

Alice Krieg-Planque et Claire Oger

\section{(2) OpenEdition}

\section{Journals}

Édition électronique

URL : https://journals.openedition.org/mots/19870

DOI : $10.4000 /$ mots. 19870

ISSN : 1960-6001

Éditeur

ENS Éditions

Édition imprimée

Date de publication : 30 novembre 2010

Pagination : 91-96

ISBN : 978-2-84788-235-3

ISSN : 0243-6450

Référence électronique

Alice Krieg-Planque et Claire Oger, « Discours institutionnels.Perspectives pour les sciences de la communication », Mots. Les langages du politique [En ligne], 94 | 2010, mis en ligne le 06 novembre 2012, consulté le 28 avril 2022. URL : http://journals.openedition.org/mots/19870 ; DOI : https:// doi.org/10.4000/mots. 19870 


\section{Mots \\ Les langages du politique}

№ 94 novembre 2010

\section{Trente ans d'étude des langages du politique (1980-2010)}

ouvrage coordonné par Paul BACOT, Marlène Coulomb-GulLY, Jean-Paul Honoré, Christian Le BART, Claire Oger, Christian Plantin

\section{SOMMAIRE}

Paul Bacot, Marlène Coulomb-Gully, Jean-Paul Honoré, Christian Le Bart, Claire Oger, Christian Plantin

Le discours politique n'est pas transparent. Permanence

et transformations d'un objet de recherche

OUTILS ET ENJEUX DU DISCOURS POLITIQUE

Ruth Amossy, Roselyne Koren

Argumentation et discours politique

Christian Plantin

Argumentation-rhétorique. Les eaux mêlées

Caroline Ollivier-Yaniv

Discours politiques, propagande, communication, manipulation 
Marc Bonhomme

La caricature politique

Paul Bacot

Développement et diversification d'une onomastique politique

Ruth Wodak

The Discursive Construction of History. Brief Considerations

Henri Boyer

Les politiques linguistiques

\section{LIEUX DE LA PRODUCTION DU DISCOURS POLITIQUE}

Christian Le Bart

Parler en politique

Dominique Maingueneau

Le discours politique et son «environnement»

Alice Krieg-Planque, Claire Oger

Discours institutionnels. Perspectives pour les sciences

de la communication

Sophie Béroud, Josette Lefèvre

Le corpus syndical. Une expérience au long cours

Corinne Gobin, Jean-Claude Deroubaix

L'analyse du discours des organisations internationales.

Un vaste champ encore peu exploré

François de la Bretèque

Le retour de la parole politique dans le cinéma français

Jean-Claude Soulages

Vie et mort du citoyen cathodique

Jacques Guilhaumou

Les discours de la Révolution française.

Aperçu d'ensemble d'un trajet de recherche (1980-2009) 


\section{DISCIPLINES ET CHAMPS DE RECHERCHE POUR L'ÉTUDE DES LANGAGES DU POLITIQUE}

Philippe Braud

L'apport de la science politique à l'étude des langages du politique

Claire Blandin

L'apport de l'histoire des médias à l'étude des langages du politique

Jean-François Tétu, Bernard Lamizet

Les SIC et les langages du politique

Sylvianne Rémi-Giraud

Sémantique lexicale et langages du politique.

Le paradoxe d'un mariage difficile?

Marlène Coulomb-Gully, Juliette Rennes

Genre, politique et analyse du discours.

Une tradition épistémologique française gender blind

Johannes Angermüller

Analyser le discours politique en Allemagne (1980-2010)

Érik Neveu

L'apport de Pierre Bourdieu à l'analyse du discours.

D'un cadre théorique à des recherches empiriques

Roselyne Ringoot

Questionner le discours avec Michel Foucault.

Actualisations théoriques et actualité éditoriale

\section{ENTRETIEN}

Maurice Tournier

Mots et politique, avant et autour de 1980

La revue Mots. Les langages du politique encourage l'usage des rectifications de l'orthographe proposées par le Conseil supérieur de la langue française et approuvées par l’Académie (Journal officiel, $n^{\circ}$ 100, 6 décembre 1990). 


\section{Alice Krieg-Planque \\ Claire Oger}

\section{Discours institutionnels. Perspectives pour les sciences de la communication}

L'analyse du discours porte un intérêt spécifique à la question de la légitimation des institutions, en insistant sur le système de contraintes qui s'exerce sur l'énonciation. Les objets de prédilection de la discipline ont d'abord été constitués par les discours d'appareils (syndicats, partis...), ou idéologiquement marqués (discours xénophobes, discours d'extrême droite, discours antiracistes...). Comme le rappelle Dominique Maingueneau (1991), l'enrichissement des cadres linguistiques de l'analyse a accompagné les premiers développements de la discipline. À mesure que l'intérêt s'est élargi aux discours médiatiques et à différents types de prise de parole dans l'espace public, l'interrogation sur la légitimité s'est enrichie de nouvelles problématiques. La collection de l'ensemble des numéros de la revue Mots. Les langages du politique atteste d'ailleurs cette évolution, tout comme un certain nombre de travaux de référence en analyse du discours, qui témoignent de la persistante diversité des objets et des méthodes (citons par exemple Hetzel, Lefèvre, Mouriaux, Tournier éd., 1998 ou Delforce, Noyer, 1999).

Mais l'analyse du discours est loin d'être le seul champ de recherche à s'être attaché à la question des mots dans les institutions. On pense bien sûr à la parenté qui unit analyse de discours et sociolinguistique (Boutet, Maingueneau, 2005) : au-delà des spécificités et des traditions de chacune (corpus et terrains, méthodes, problématiques...), leurs coopérations ont construit un domaine commun, celui des approches langagières du social. Les livraisons successives de la revue Langage et société fournissent une illustration de ce rapprochement.

S'agissant du fait institutionnel, on peut également, pour appréhender les phénomènes discursifs, s'appuyer sur des études en anthropologie (Abélès et al., 1999), en sociologie (Bereni, Jaunait, 2009), en science politique (Cusso, Gobin, 2008), en sciences de l'information et de la communication (Huët, 
2008), ou encore sur les publications de collectifs transdisciplinaires tels que le réseau Langage \& Travail (Borzeix, Fraenkel, 2001). Là aussi, les contraintes qui régissent les activités sociales sont mises en avant, qu'il s'agisse de souligner l'imposition d'un ordre institutionnel ou l'élaboration d'une culture commune.

La question de la constitution des corpus a encore enrichi ces échanges, en permettant une réflexion approfondie sur la diversité des textes qui pouvaient être réunis dans des corpus hétérogènes, assez différents des séries régulières et homogènes que privilégiait l'analyse du discours à ses débuts. Les SIC ont contribué à répandre la pratique d'études menées sur une pluralité de documents rassemblés autour d'un objet de recherche, permettant par exemple de s'intéresser à des «écrits professionnels» ou à des «écrits au travail» de plusieurs natures (Delcambre, 2000). La fréquentation des sociologues a conduit quant à elle à se tourner également vers l'analyse de la parole recueillie en entretien, retravaillant ainsi sans la dénier la frontière, fondatrice de l'analyse du discours, entre parole et discours (Oger, 2003), tandis que d'autres démarches rapprochent archives historiques, productions institutionnelles et entretiens (Fiala éd., 1999).

Seules les relations avec le droit sont aujourd'hui trop rares, tant la réflexion sur le discours juridique reste cantonnée dans le cercle des spécialistes de la sémiotique juridique ou de l'histoire du droit. Il n'en reste pas moins que cette direction de travail mériterait bien des explorations pour les analystes du discours, comme l'attestent des travaux encore isolés et davantage centrés sur les phénomènes de médiatisation (Thomas, 2008).

Nous prendrons ici le parti de négliger les appartenances disciplinaires pour envisager l'enrichissement que suggère, pour les sciences de l'information et de la communication (SIC), l'ensemble de ces recherches. Celles-ci permettent en effet de dégager deux aspects qui nous semblent essentiels à la compréhension des discours institutionnels, et qui peuvent également contribuer au développement d'un programme de travail en SIC. Le premier aspect relève d'une stabilisation des énoncés. Le second procède d'un effacement de la conflictualité. Ce double principe de formation des discours institutionnels, qui sont avant tout des discours autorisés, est repérable dès les premiers travaux d'analyse du discours, qui débusquent les fonctionnements de la phraséologie et de la « langue de bois» (Fiala, Pineira, Sériot éd., 1989). Il est possible aujourd’hui de reformuler les termes de ces problématiques et d'en reposer les enjeux.

Concernant le premier aspect, on observe que la production des discours institutionnels, tout autant que la circulation des énoncés dans l'espace public, obéissent à des régularités qui réduisent la diversité des énoncés possibles. Cette stabilisation peut se manifester, au niveau lexical, par des phénomènes de formulations conventionnelles, de sloganisation, de figement, de collocation, de cooccurrence, dont les formules (Krieg-Planque, 2009) fournissent des illustrations privilégiées. «Développement durable », «mondialisation », « économie de la connaissance» sont autant d'expressions qui combinent figement 
formel et indétermination du référent. De même, la prolifération de la formule "purification ethnique» et de ses variantes, à propos du conflit yougoslave, est directement liée à la construction hétérogène, voire polémique, des référents désignés (Krieg-Planque, 2003).

La raréfaction des énoncés possibles recouvre également des phénomènes génériques et textuels liés à l'organisation du discours, aux modalités de l'argumentation légitime ou à des formats rédactionnels. Le compte rendu analytique des débats à l'ONU (Duchêne, 2004), ou le signalement d'enfant en danger (Serre, 2009) matérialisent des formes routinisées de l'écriture en contexte contraint et portent la trace des enjeux sociopolitiques et institutionnels qu'ils engagent. Sur de tels genres bien identifiables, un certain nombre de travaux apportent un éclairage plus spécifiquement linguistique, explorant l'expression des tensions et des conflits qui en sont constitutifs (Cislaru, PugnièreSaavedra, Sitri, 2008). En cela, ils rejoignent l'analyse du deuxième trait que nous avons souhaité présenter : l'effacement de la conflictualité.

En effet, dans l'institution, les locuteurs, sommés de répondre à des exigences complexes voire contradictoires, obéissent à une injonction plus générale : organiser la polyphonie, réduire la dissonance, produire la cohérence. Dans ce mouvement, ils tendent à effacer les traces de la conflictualité et des tensions internes (Oger, Ollivier-Yaniv, 2006). C'est ainsi également qu'ils peuvent opérer la neutralisation de l'opposition entre posture d'expert et positionnement politique, la conciliation des intérêts entre partenaires publics et privés, la dénégation des divergences d'opinion, ou encore la légitimation du discours savant (sur ce dernier point, voir Maris éd., 2002).

Ces fonctionnements discursifs s'observent d'ailleurs à différentes échelles. Ainsi, la formule «développement durable » (Krieg-Planque, à paraître) semble résoudre une contradiction entre impératif de croissance et exigence de préservation de l'environnement, au prix de l'effacement d'une opération concessive, pourtant constitutive de l'expression (dans des formulations du type «favoriser le développement tout en préservant... »). Dans un autre ordre d'idées, les discours des jurys de concours permettent de dégager, dans les épreuves de culture générale, des modèles d'expression sous-tendus aussi bien par des changements d'orientation argumentative (le fameux «balancement circonspect» des énarques) que par des injonctions contradictoires qui enserrent l'ethos imposé au candidat dans un réseau complexe d'obligations (Oger, 2008). On trouvera dans les travaux que nous citons ici d'autres illustrations de ces formes de «lissage » et de leurs manifestations concrètes. Par exemple, le discours de syndicalistes européens, recueilli par entretiens, mêle les registres du militantisme et de l'expertise, pourtant présentés comme antagonistes dans d'autres contextes. Le monde de l'entreprise quant à lui ignore bien souvent la distance qui sépare travail prescrit et travail réel, et soumet volontiers les acteurs à des formes de communication paradoxale. 
La sphère politique elle-même tend aujourd'hui à effacer la diversité des catégories d'acteurs, et à réduire le caractère disparate de leurs mobiles et de leurs valeurs, au profit de la promotion de la "gouvernance», à l'instar des organisations internationales qui ont contribué à légitimer ce mot d'ordre (voir la contribution de Jean-Claude Deroubaix et Corinne Gobin dans le présent volume). La faveur que connaît la «démocratie participative », ou le rôle croissant auquel est conviée la "société civile», s’inscrivent dans le même mouvement. Ce qu'il est convenu d'appeler la «dépolitisation » est une des réalisations les plus caractéristiques de l'effacement des conflits en discours. Elle fournit le soubassement d'une légitimité nouvelle qui vient compléter et concurrencer la célébration de l’intérêt général dans les institutions publiques et la rhétorique de l'universalisme dans les organisations internationales (Cusso, Gobin, 2008; Rist, 2002).

Comme on le voit dans ce dernier paragraphe, le «parler neutre» fait une large place au «style formulaire » : nous retrouvons ici les figements qui rendent les productions institutionnelles aussi largement prévisibles. Les deux dynamiques de formation des discours autorisés, stabilisation des énoncés et effacement de la conflictualité, apparaissent comme complémentaires. Par l'effet d'évidence et de reconnaissance du préconstruit, la formule appelle l'assentiment, engage à l'adhésion, décourage la contradiction. Par là, elle inhibe les contre-discours possibles et tend à escamoter les occasions d'affrontement. Symétriquement, la minoration des désaccords et des conflits, la valorisation du consensus, s'accomplissent plus aisément par le truchement de formulations stéréotypées, de tournures convenues.

Ence sens, les apports de l'analyse des discours institutionnels ne s'adressent pas uniquement aux spécialistes des institutions publiques. Ils concernent aussi les chercheurs qui s'intéressent aux entreprises et aux organisations, au sens où elles sont soumises au même impératif de légitimation. Soucieuses de ménager l'adhésion à leur projet, tant en matière de communication externe qu'interne, elles recourent aux deux principales ressources que nous venons de présenter et qui caractérisent selon nous les discours autorisés. Nous n'en prendrons que deux exemples pour conclure.

Les supports et les «outils de gestion» qui entendent mettre en œuvre la «responsabilité sociale de l'entreprise» appellent une étude des énoncés, des genres ou des opérations argumentatives qui contribuent à transformer les arènes publiques et à brouiller la frontière entre pouvoirs publics et acteurs privés. Dans un domaine voisin, les appels à la représentation de la «diversité» (dans les entreprises, les concours, sur la scène médiatique, politique...) ont alimenté une prolifération d'emplois formulaires («issus de la diversité », "charte de la diversité », " promotion de la diversité »...), et dans le même temps, dissuadé les contradicteurs potentiels. Nous avons affaire ici à l'un de ces «discours sans adversaires» que Philippe Juhem (2001) met en 
évidence à propos des organisations humanitaires. En ce sens, l'analyse des discours institutionnels ne nous invite nullement à établir une discontinuité entre institutions, entreprises et associations, mais bien plutôt à considérer ce qui caractérise tout "groupement social légitimé» (Douglas, 1999) du point de vue de la production des discours et de la communication.

Ainsi les chercheurs en SIC peuvent y voir une perspective qui, sans les dessaisir de leurs objets, les éclaire différemment. Si les origines et les premiers développements de la discipline se sont focalisés sur l'univers médiatique, et si l'intérêt pour les questions politiques ne s'est développé que plus tardivement (voir ici même l'article de Jean-François Tétu et Bernard Lamizet), cette restriction est loin d'épuiser le champ des pratiques communicationnelles, de la circulation des discours, et des formes d'imposition de pouvoir qui s'y réalisent. De tels objets invitent à se saisir de l'ambition critique qui anime l'analyse du discours depuis ses débuts, et à rendre compte à la fois des mutations de la communication et de la permanence d'enjeux symboliques. En faisant apparaître la dynamique de production des discours autorisés qui investissent les arènes publiques, l'analyse des discours institutionnels peut contribuer à préciser un programme de travail qui concerne la plupart des phénomènes communicationnels, dès lors que l'on se place à l'échelle sociale.

\section{Références}

AbÉLÈs M. et al. éd., 1999, Ethnologie française, vol. 29, n 4 , Les mots des institutions. BerEnI L., Jaunait A. éd., 2009, Raisons politiques, n³ 35, Usages de la diversité.

Borzeix A., Fraenkel B. éd., 2001, Langage et travail. Communication, cognition, action, Paris, CNRS (Communication).

Boutet J., Maingueneau D., 2005, "Sociolinguistique et analyse de discours. Façons de dire, façons de faire », Langage et société, nº114, p.15-47.

Cislaru G., Pugnière-SaAvedra F., Sitri F. éd., 2008, Les Carnets du Cediscor, no ${ }^{10}$, Analyse de discours et demande sociale. Le cas des écrits de signalement.

Cusso R., Gobin C. éd., 2008, Mots. Les langages du politique, n88, novembre, Du discours politique au discours expert.

DELCAMBRE P., 2000, «Quels corpus pour la recherche sur les pratiques de communication dans les entreprises et les organisations? ", Sciences de la société, nº 50-51, p. 69-85.

DelforCe B., Noyer J., 1999, «Pour une approche interdisciplinaire des phénomènes de médiatisation. Constructivisme et discursivité sociale», Études de communication. Techniques d'expression, information, communication, nº 22, p.13-39.

Douglas M., 1999 [1986], Comment pensent les institutions, Paris, La Découverte / MAUSS (Recherches).

DUCHÊNE A., 2004, «Construction institutionnelle des discours. Idéologies et pratiques dans une organisation supranationale », TRANEL (Travaux neuchâtelois de linguistique), $\mathrm{n}^{\circ} 40$, p. 93-115. 
Fiala Pierre éd., 1999, In/égalité/s. Usages lexicaux et variations discursives (18e-20 siècles), Paris, L'Harmattan (Sémantiques).

Fiala P., Pineira C., SÉriot P. éd., 1989, Mots. Les langages du politique, n²1, décembre, Langues de bois?.

Hetzel A.-M., LefèVre J., Mouriaux R., Tournier M. éd., 1998, Le syndicalisme à mots découverts. Dictionnaire des fréquences (1971-1990), Paris, Syllepse.

HUËT R., 2008, Les dynamiques sociales entre acteurs ou organisations lors des processus d'engagement. Le cas des chartes et codes de conduite, thèse de doctorat en sciences de l'information et de la communication, Université Charles de Gaulle Lille 3, sous la direction de Pierre Delcambre.

JUHEM Ph., 2001, "La logique du succès des énoncés humanitaires. Un discours sans adversaires", Mots. Les langages du politique, $\mathrm{n}^{\circ} 65$, mars, p. 9-27.

KrIeg-PlAnque A., 2003, "Purification ethnique». Une formule et son histoire, Paris, CNRS (Communication).

- 2009, La notion de «formule » en analyse du discours. Cadre théorique et méthodologique, Besançon, Presses universitaires de Franche-Comté.

- À paraître (2011), «La formule "développement durable”. Un opérateur de neutralisation de la conflictualité», Langage et société, nº 135.

MaIngueneAu D., 1991, L'analyse du discours. Introduction aux lectures de l'archive, Paris, Hachette Supérieur (Linguistique).

MARIS B. éd., 2002, Sciences de la société, n055, La légitimation du discours économique.

Oger C., Ollivier-Yaniv C., 2006, «Conjurer le désordre discursif. Les procédés de “lissage" dans la fabrication du discours institutionnel», Mots. Les langages du politique, $n^{\circ} 81$, juillet, p. 63-77.

OGER C., 2003, «Communication et contrôle de la parole. De la clôture à la mise en scène de l'institution militaire», Quaderni. La revue de la communication, no 52 , p. 77-92.

- 2008, Le façonnage des élites de la République. Culture générale et haute fonction publique, Paris, Presses de Sciences Po (Académique - Fait politique).

RIST G. éd., 2002, Nouveaux cahiers de l'IUED, n013, Les mots du pouvoir. Sens et nonsens de la rhétorique internationale.

SERRE D., 2009, Les coulisses de l'État social. Enquête sur les signalements d'enfant en danger, Paris, Liber / Raisons d'Agir (Cours et travaux).

Thomas C., 2008, Le bruit de la loi. Comment les lois deviennent médiatiques, thèse de doctorat en sciences sociales, ENS Cachan, sous la direction de Patrice Duran. 